\title{
Eisenmenger syndrome in an adult patient with a large patent ductus arteriosus
}

\author{
Konstantinos Dimopoulos
}

Affiliations: Adult Congenital Heart Centre and Centre for Pulmonary Hypertension, NIHR Cardiovascular Biomedical Research Unit, Royal Brompton Hospital and the National Heart and Lung Institute, Imperial College, London, UK.

Correspondence: K. Dimopoulos, Adult Congenital Heart Centre and Centre for Pulmonary Hypertension, Royal Brompton Hospital, Sydney Street, London, SW3 6NP, UK. E-mail: k.dimopoulos02Agmail.com

ABSTRACT This is the case of a young female who was seen at our adult congenital heart disease and pulmonary hypertension service (Royal Brompton Hospital, London, UK) at the age of 17 years. She initially presented at the age of 4 years with increasing shortness of breath. At that time, there was differential cyanosis with clubbing and lower oxygen saturations in the toes (82\%) compared with her fingers $(95 \%)$. On echocardiography there was evidence of severe pulmonary hypertension and a large patent ductus arteriosus (PDA) with low velocity bidirectional shunting. She underwent cardiac catheterisation at the time which showed a pulmonary arterial pressure equal to that of the aorta.

0 @ERSpublications

$\mathrm{PH}$ is not rare in CHD patients and is best treated in centres combining CHD/PH expertise http://ow.ly/psfQz

\section{Question 1}

What is the diagnosis and would you close this PDA?

The patient had a bidirectional shunt (thus also shunted right-to-left) at PDA level and had severe pulmonary arterial hypertension (PAH) and had, therefore, developed Eisenmenger syndrome (fig. 1) [1]. $\mathrm{PAH}$ related to congenital heart disease (CHD) can be classified into four groups (table 1) according to the severity of the disease and the presence/size of the defect. Eisenmenger syndrome is at the extreme end with regards to the severity of PAH and the degree of cyanosis [2]. While Eisenmenger patients have severe pulmonary vascular disease, with some following an aggressive course in childhood, patients reaching adulthood may remain fairly stable for years or decades and have a prognosis that is better compared to those with $\mathrm{PAH}$ after repair of $\mathrm{CHD}$ and those with severe PAH in the presence of a small defect [3-5].

It is clear that this defect should be left open once significant pulmonary vascular disease with reversal of the shunt through the PDA has developed. Even though the PDA is responsible for the onset of PAH it is now acts as a "relief valve", offloading the pressure overloaded right ventricle. While closure of the PDA can easily be achieved percutaneously, this can lead to right ventricle failure hours, weeks, months or years after the procedure, adversely affecting outcome. The same applies for patients with Eisenmenger syndrome in the presence of an atrial or ventricular septal defect.

Our patient presented with a typical Eisenmenger phenotype early in life. The full Eisenmenger phenotype with right-to-left shunting and overt cyanosis typically becomes manifest later in childhood or in

Received: Sept 192013 | Accepted after revision: Sept 292013

Conflict of interest: Disclosures can be found alongside the online version of this article at err.ersjournals.com

Provenance: Publication of this peer-reviewed article was supported by Actelion Pharmaceuticals Ltd, Switzerland (principal sponsor, European Respiratory Review issue 130).

Copyright OERS 2013. ERR articles are open access and distributed under the terms of the Creative Commons Attribution Non-Commercial Licence 3.0 

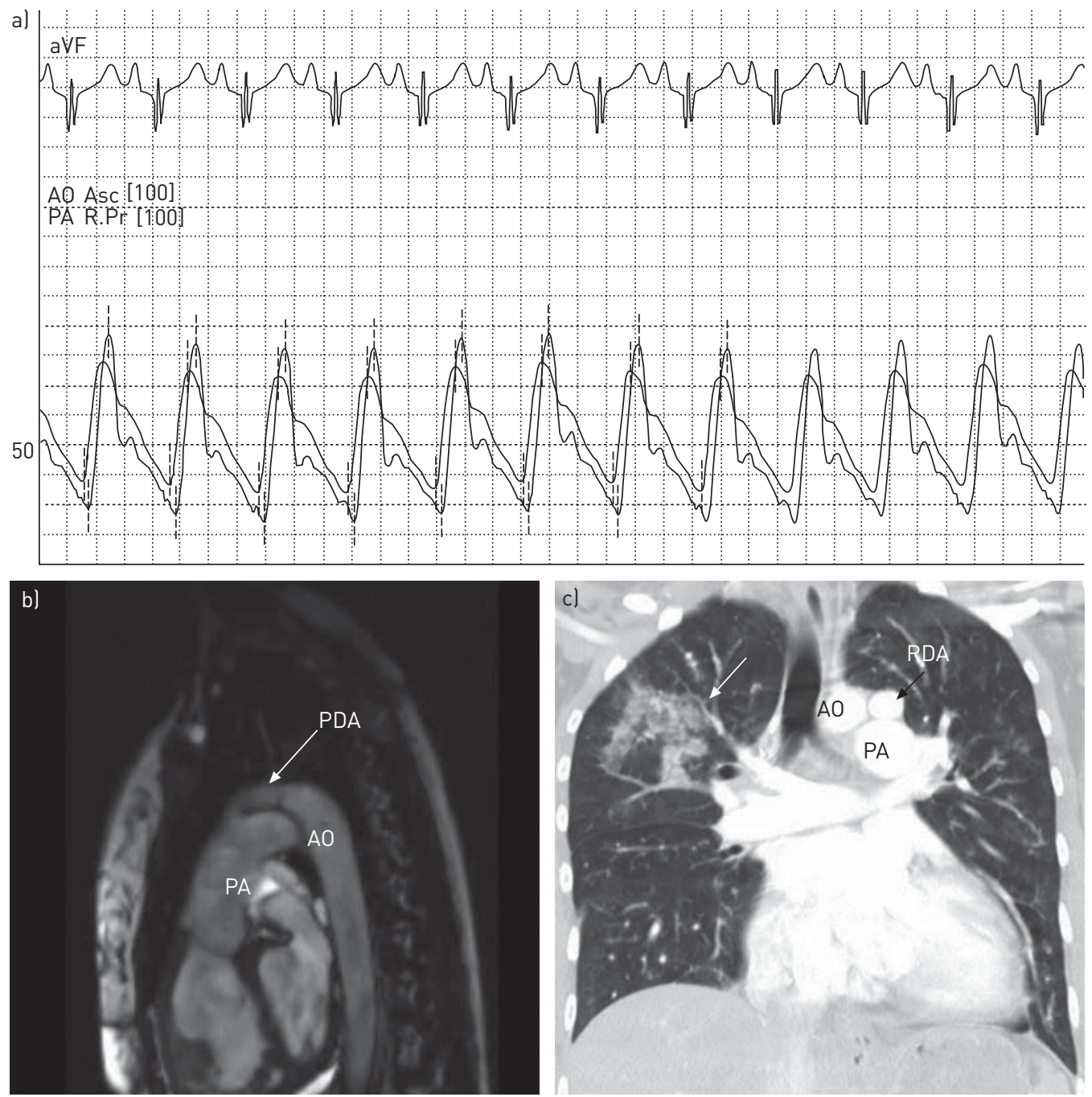

FIGURE 1 a) Pressure tracings from the aorta $(\mathrm{AO})$ and pulmonary artery (PA), demonstrating systemic levels of pulmonary arterial pressures. Mean pulmonary arterial pressure is $54 \mathrm{mmHg}$. b) Cardiac magnetic resonance showing the large patent ductus arteriosus (PDA), with bidirectional shunting between the PA to the descending AO. c) Computed tomography of the thorax demonstrating an area of intrapulmonary haemorrhage in the left upper lung (white arrow). The PDA is also seen between the AO and PA (black arrow).

\section{TABLE 1 Types of pulmonary arterial hypertension (PAH) in congenital heart disease}

\section{A. Eisenmenger syndrome}

Includes all systemic-to-pulmonary shunts due to large defects leading to a severe increase in PVR and a reversed (pulmonary-to-systemic) or bidirectional shunt. Cyanosis, erythrocytosis, and multiple organ involvement are present

\section{B. PAH associated with systemic-to-pulmonary shunts}

Patients with moderate-to-large defects, in which the increase in PVR is mild-to-moderate, left-to-right shunt is still largely present and no cyanosis is present at rest

\section{PAH with small defects}

Patients with a clinical picture very similar to idiopathic PAH, who have (coincidental?) small cardiac defects: a ventricular septal defect $<1 \mathrm{~cm}$ and an atrial septal defect $<2 \mathrm{~cm}$

\section{PAH after corrective cardiac surgery}

Congenital heart disease has been corrected but PAH is still present immediately after surgery, or has recurred several months or years after surgery in the absence of significant post-operative residual congenital lesions or defects that originate as sequelae of previous surgery

PVR: pulmonary vascular resistance. Reproduced with modification from [2]. 
adolescence/early adult life. The reason for this heterogeneity in presentation in patients with similar cardiac anatomy remains unknown and has been attributed to different susceptibility of the pulmonary vasculature and different response to the haemodynamic insult.

At the time of diagnosis of PAH (in childhood), no oral PAH therapies were available. Thus, the patient was started on nifedipine, a calcium channel blocker.

\section{Question 2}

Are calcium channel blockers indicated in Eisenmenger syndrome?

According to available evidence and current guidelines, patients with idiopathic PAH should be assessed for reversibility at the time of initial cardiac catheterisation and put on high dose of calcium channel blockers when an adequate response to acute pulmonary vasodilator administration is seen [2]. The criteria for defining vasoreactivity in idiopathic $\mathrm{PAH}$ (decrease in mean pulmonary arterial pressure $\geqslant 10 \mathrm{mmHg}$ to an absolute value $\leqslant 40 \mathrm{mmHg}$ ) do not apply here. No data are available on the usefulness of long-term calcium channel blocker therapy in Eisenmenger patients. Minor vasoreactivity can even be observed in Eisenmenger patients and has been reported to predict outcome [6]. While there is little value in performing vasoreactivity testing in a CHD-PAH patient for the purpose of assessing eligibility for calcium channel blocker therapy, reversibility studies are often performed in patients with CHD and borderline pulmonary vascular resistance to assess operability [7-9]. The two indications should not be confused and the criteria for defining vasoreactivity differ. Evidence-based criteria for assessing operability are still lacking and the decision to operate is best taken in experienced centres, based on the available clinical information (including catheterisation data).

The patient was first seen in the adult congenital heart disease service at the age of 17 years. On examination, blood pressure and heart rate were normal. She did not appear to have cyanosis in her upper body. She had no finger clubbing, but significant toe clubbing. Oxygen saturations in her fingers were normal, but low in the toes $(83 \%)$. There were no signs of congestive heart failure. On auscultation, there was a normal first, but prominent second heart sound and a mild holosystolic murmur over the left lower sternal border. There was no murmur over the PDA.

\section{Question 3}

Why is there no murmur generated by the PDA?

A large PDA generates an audible murmur as long as there is a sufficient pressure gradient between the aorta and the pulmonary artery to sustain a high velocity shunt. As pulmonary vascular disease progresses, the pressure difference between the great vessels falls progressively, and so does the intensity of the murmur. In patients with Eisenmenger syndrome, the shunt is bidirectional, mainly from right-to-left, low-velocity and with no associated murmur. A soft holosystolic murmur in the tricuspid area and a long diastolic murmur in the pulmonary areas may be heard if tricuspid and pulmonary regurgitation, respectively, develop. The same applies for patients with Eisenmenger syndrome in the presence of an atrial or ventricular septal defect.

In the absence of a high velocity shunt at PDA level in Eisenmenger patients it may be difficult to diagnose a PDA on echocardiography. In fact, Eisenmenger patients with a PDA can, at times, be mislabelled as idiopathic PAH. All patients with a new diagnosis of PAH should be investigated for differential cyanosis and peripheral clubbing, especially when erythrocytosis is present (see below), and the presence of the PDA confirmed on computed tomography of the pulmonary arteries or CMRI (fig. 1b).

When the patient was seen in the adult service, blood testing was performed demonstrating an increase in haemoglobin concentration $\left(18.4 \mathrm{~g} \cdot \mathrm{dL}^{-1}\right)$ and haematocrit $(56 \%)$, with evidence of iron deficiency (ferritin $10 \mu \mathrm{g} \cdot \mathrm{L}^{-1}$ and transferrin saturation $\left.13 \%\right)$. Mean cellular volume was normal. Brain natriuretic peptide (BNP) was raised at $40 \mathrm{pmol} \cdot \mathrm{L}^{-1}\left(\right.$ normal range $\left.<4 \mathrm{pmol} \cdot \mathrm{L}^{-1}\right)$.

\section{Question 4}

Why is haemoglobin concentration raised, does the patient require venesection and what is the significance of a raised BNP in Eisenmenger patients?

An increase in haemoglobin levels in patients with cyanotic heart disease is a welcome compensatory mechanism, aimed at increasing the blood's oxygen carrying capacity. It is best referred to as "erythrocytosis" to distinguish it from polycythaemia rubra vera, with which it does not share the same propensity to thrombotic and embolic events [10]. Erythrocytosis in cyanotic patients is often associated with a decrease in platelet count and, at times, white cell count. Even though no validated algorithms exist to establish the appropriate haemoglobin concentration for individual patients, in the presence of systemic oxygen saturations of $80-85 \%$, haemoglobin of $19-24 \mathrm{~g} \cdot \mathrm{dL}^{-1}$ is usually observed $[5,11,12]$. 
Iron deficiency is common in cyanotic heart disease, and is encountered in up to one-third of patients [5, 13-15]. It is multifactorial, probably due to increased iron requirement, decreased absorption, bleeding, etc. Iron deficiency is an adverse prognostic marker in this population [14]. All cyanotic patients should be regularly screened for iron deficiency using transferrin receptor levels or, when not available, ferritin and transferrin saturations. A low mean cellular volume is not commonly encountered in iron deficient cyanotic patients and should not be used to exclude iron deficiency [16].

Venesections are best avoided in cyanotic CHD patients as they promote iron deficiency and can lead to cerebrovascular events, probably due to a reduction in oxygen delivery to the brain $[10,17]$. Venesections should only be performed in patients with severe hyperviscosity symptoms in the presence of very high haematocrit $(>65 \%)$ and in the absence of dehydration and iron deficiency. In fact, severe iron deficiency shares symptomatology with hyperviscosity. Adequate fluid replacement is essential, using filters to avoid air emboli.

Iron supplementation is essential in patients who are found to be iron deficient. A single centre, nonrandomised cohort study on cyanotic patients with CHD showed that iron supplementation alone can improve exercise capacity and quality of life. No cases of excessive erythropoiesis or new-onset severe hyperviscosity symptoms were seen and no cases required venesection [18].

Elevated BNP concentrations are common in patients with Eisenmenger syndrome and relate to a higher risk of death. A cut-off BNP value of $100 \mathrm{pg} \cdot \mathrm{mL}^{-1}\left(\sim 30 \mathrm{pmol} \cdot \mathrm{L}^{-1}\right)$ has been suggested as a marker of adverse prognosis. Increases in BNP concentration over time have also been found to be of prognostic significance. Thus, BNP can be a useful adjunct in guiding therapy in Eisenmenger syndrome [19].

When questioned about exercise limitation, the patient insisted that she was not significantly limited in her ordinary activities. However, when looking into specific activities, it became evident that she could only manage $\sim 20$ min walking on the flat or one flight of stairs before becoming very short of breath. She found activities such as gardening and vacuuming difficult and, hence, avoided them. She achieved a distance of only $340 \mathrm{~m}$ on the 6 -min walk test (6MWT), with a significant drop in oxygen saturation to $70 \%$ in the toe.

\section{Question 5}

What is the patient's current functional class?

The pulmonary hypertension guidelines suggest that only Eisenmenger patients in functional class 3 should be considered for treatment with advanced therapies for PAH [2]. However, assessing the functional class in $\mathrm{PAH}$ patients with CHD can be difficult using the New York Heart Association or World Health Organization classification. Onset of symptoms early in life can modify symptom perception and lead to chronic adaptation of everyday "ordinary" activities, as opposed to patients who develop exercise limitation later in life and over a shorter period of time (e.g. those with idiopathic PAH) [15, 20, 21]. In order to avoid underestimating their degree of limitation, patients should be asked specific questions on typical ordinary activities (walking, climbing stairs, showering, cleaning, vacuuming, gardening, dancing, etc.) and specific landmarks should be explored (e.g. distance from local train/metro station, distance from hospital entrance or cardiology department) on a regular basis [22]. Addition of the above information to indices of objective exercise capacity (exercise testing), imaging and BNP should influence the decision as to whether to initiate therapies $[23,24]$.

The 6MWT is commonly used for assessing exercise capacity and response to therapies in Eisenmenger patients. In a recent, single centre study on Eisenmenger patients, 6MWT distance (but not the rate of change of 6MWT distance) was a strong predictor of outcome, while functional class seemed to lack the reproducibility and discriminative power to follow subtle changes over time [23]. It is noteworthy that a lack of predictive power of 6-min walk distance change on long-term outcomes is also seen in other forms of PAH.

Nifedipine was stopped and the patient underwent iron supplementation. Thereafter she was initiated on the endothelin receptor antagonist (ERA) bosentan [18]. 4 months after the introduction of bosentan, an increase of $60 \mathrm{~m}$ in 6-min walk distance was observed.

\section{Question 6}

What is the evidence for the use of advanced therapies for PAH in Eisenmenger patients?

The safety and efficacy of the ERA bosentan was established by the BREATHE-5 trial [25]. This was a 16 -week randomised controlled trial on Eisenmenger patients in functional class 3 with a ventricular septal defect or large atrial septal defect, which reported a statistically significant beneficial effect on 6MWT distance and pulmonary vascular resistance [25]. It also reported an improvement in functional class but no deterioration in systemic oxygen saturations [25]. It is also noteworthy that, on average, the 6MWT distance and pulmonary vascular resistance worsened in the placebo group, suggesting progression of the disease 
over the relatively short duration of the study. The effect on 6MWT distance was maintained at 12 months on the open-label extension and this improvement was confirmed in both patients with a ventricular septal defect and an atrial septal defect $[26,27]$. Further small trials have demonstrated the efficacy and safety of sildenafil and tadalafil in this setting [28-30]. The long-term efficacy of advanced therapies in Eisenmenger patients has now been confirmed in large registries [31]. There is also evidence of a beneficial effect of advanced therapies on survival from a single centre retrospective study [32].

Data are still lacking on the optimal mode of following Eisenmenger patients in terms of assessing the need for starting therapies, their efficacy and the need to up-titrate or escalate (combination therapy). In clinical practice, functional status, 6MWT distance, echocardiographic parameters and BNP are commonly used, but specific achievement targets have not yet been established in this population [5, 23, 32]. Moreover, the value of repeat cardiac catheterisation as a follow-up tool remains controversial. Cardiac output in Eisenmenger patients is maintained through right-to-left shunting and is unlikely to carry the same prognostic power as in idiopathic PAH. Moreover, calculation of pulmonary vascular resistance is difficult in patients with a PDA and those with complex cardiac anatomy.

A large proportion of Eisenmenger patients have Down syndrome. In these patients, neither subjective measures of effort tolerance nor formal exercise testing are possible and can be inaccurate [33, 34]. An improvement in quality of life is probably the most important target for therapy in all Eisenmenger patients, especially those with Down syndrome.

\section{Question 7}

What other supportive measures should be taken in Eisenmenger patients?

Supportive measures are as important in Eisenmenger syndrome as advanced therapies [15]. These mainly consist of avoiding pitfalls, such as submitting patients to non-essential general anaesthesia or sedation, which carry significant risks. Venesection should be avoided and iron deficiency treated [10]. Dehydration should be avoided. Patients should remain physically active while avoiding strenuous efforts, competitive sports and extreme isometric efforts. There is little evidence to support long-term supplemental oxygen use. Endocarditis prophylaxis is still advocated by international guidelines, in view of the increased risk of embolic events, especially cerebral abscess [35]. Regular dental review is essential.

Acute arrhythmias, both ventricular and supraventricular, can lead to rapid deterioration and should be managed promptly. Electrical cardioversion is often required and is best performed under the care of senior anaesthetists experienced in PAH and Eisenmenger syndrome.

Haemoptysis is common in Eisenmenger patients and, in most cases, is self-limiting; however, massive haemoptysis can lead to death (fig. 1c) [36]. When assessing a patient with haemoptysis, evaluation of the airway, breathing and circulation is the first step. Bleeding cessation can be achieved through interruption and/or reversal of anticoagulation. In massive haemoptysis, protection of airways, fluid resuscitation and anti-fibrinolytic drugs (e.g. tranexamic acid) may be used with caution. Underlying lung infection should be treated promptly. Haemoglobin levels should be corrected to levels appropriate for the oxygen saturations and iron should be supplemented. Strict in-hospital observation is recommended. Escalation of advanced therapies should be considered.

There is still controversy on the use of anticoagulants in Eisenmenger syndrome, with no convincing evidence for or against their use [37]. Patients with arrhythmias, in situ thrombosis of the pulmonary arteries, embolic phenomena or congestive heart failure should receive anticoagulation, in the absence of contraindications.

\section{Question 8}

Is it safe for patients with Eisenmenger syndrome to become pregnant?

The issue of pregnancy should be discussed as early as possible in all patients with pulmonary hypertension in view of the very high associated mortality (prohibitive) [38]. In a systematic review of the literature, the risk of death due to pregnancy in patients with $\mathrm{PAH}-\mathrm{CHD}$ was $\sim 30 \%$ [5]. There are also significant risks to the baby as there is an increased likelihood of prematurity and growth retardation $(\sim 80 \%)$, with an increased associated risk of long-term neurological damage. Spontaneous abortion is not uncommon, and relates to the degree of peripheral desaturation.

Even interruption of pregnancy carries risks in Eisenmenger patients, with risks of bleeding and the risk of general anaesthesia when surgical interruption is required. Appropriate contraception with progesterone only compounds, combined with barrier methods (dual contraception, especially in patients on bosentan due to possible pharmacological interaction with oral contraceptives), is therefore paramount. 


\section{Question 9}

The patient has improved significantly on advanced therapy; can I now close the defect?

Defects should never be closed in Eisenmenger patients, regardless of the magnitude of response to medical therapy. Closure of the defect risks transforming Eisenmenger physiology into an idiopathic PAH-like condition, with more rapid right ventricular dilatation and progressive dysfunction, negatively affecting long-term prognosis [3, 7]. Even for CHD-PAH patients with milder forms of pulmonary vascular disease, the long-term outcome of a "treat and repair" approach (repairing the defect after optimal response to medical therapy) has not been established and, thus, should be avoided until proven safe and effective [7].

\section{Question 10}

\section{Can Eisenmenger patients fly?}

Concerns about cyanotic CHD patients becoming unwell when flying, due to the ambient oxygen concentration being lower than at sea level, are not supported by evidence. One retrospective study questioning Eisenmenger patients on previous air travel demonstrated that most patients do not encounter significant problems and supplemental oxygen is not generally required, especially for shorter flights [39]. However, definitive evidence is lacking.

\section{Conclusions}

$\mathrm{PAH}$ is common in patients with CHD and a congenital defect should always be sought and excluded in all patients presenting with PAH. Echocardiography (with or without contrast injection) is pivotal in excluding intracardiac defects, but even simple measures, such as careful measurement of oxygen saturations in the fingers and toes at rest and after exercise, as well as serial sampling of oxygen saturation from the high superior vena cava to the pulmonary artery during cardiac catheterisation, can aid in the diagnosis of "occult" CHD. Such patients are best treated in specialist centres combining CHD and PAH expertise, as their physiology and natural history differs significantly to other types of PAH.

\section{Acknowledgements}

I would like to thank R. Doyle (Elements Communications Ltd, Westerham, UK) for medical writing support, funded by Actelion Pharmaceuticals Ltd (Allschwil, Switzerland), D. Wahl and R. Zimmerman (Actelion Pharmaceuticals Ltd) for their useful review of this article.

\section{References}

$1 \quad$ Wood P. The Eisenmenger syndrome or pulmonary hypertension with reversed central shunt. I. Br Med J 1958; 2 : 701-709.

2 Galiè N, Hoeper MM, Humbert M, et al. Guidelines for the diagnosis and treatment of pulmonary hypertension. Eur Respir J 2009; 34: 1219-1263.

3 Manes A, Palazzini M, Leci E, et al. Current era survival of patients with pulmonary arterial hypertension associated with congenital heart disease: a comparison between clinical subgroups. Eur Heart J 2013 [in press DOI: 10.1093/ eurhearti/eht072].

4 Hislop AA, Moledina S, Foster H, et al. Long-term efficacy of bosentan in treatment of pulmonary arterial hypertension in children. Eur Respir J 2011; 38: 70-77.

5 Diller GP, Dimopoulos K, Broberg CS, et al. Presentation, survival prospects, and predictors of death in Eisenmenger syndrome: a combined retrospective and case-control study. Eur Heart J 2006; 27: 1737-1742.

6 D'Alto M, Romeo E, Argiento P, et al. Pulmonary vasoreactivity predicts long-term outcome in patients with Eisenmenger syndrome receiving bosentan therapy. Heart 2010; 96: 1475-1479.

7 Dimopoulos K, Peset A, Gatzoulis MA. Evaluating operability in adults with congenital heart disease and the role of pretreatment with targeted pulmonary arterial hypertension therapy. Int J Cardiol 2008; 129: 163-171.

8 Warnes CA, Williams RG, Bashore TM, et al. ACC/AHA 2008 guidelines for the management of adults with congenital heart disease: executive summary. Circulation 2008; 118: 2395-2451.

9 Baumgartner H, Bonhoeffer P, De Groot NMS, et al. ESC Guidelines for the management of grown-up congenital heart disease (new version 2010). Eur Heart J 2010; 31: 2915-2957.

10 Spence MS, Balaratnam MS, Gatzoulis MA. Clinical update: cyanotic adult congenital heart disease. Lancet 2007; 370: $1530-1532$.

11 Broberg CS, Bax BE, Okonko DO, et al. Blood viscosity and its relationship to iron deficiency, symptoms, and exercise capacity in adults with cyanotic congenital heart disease. J Am Coll Cardiol 2006; 48: 356-365.

12 Broberg CS, Jayaweera AR, Diller GP, et al. Seeking optimal relation between oxygen saturation and hemoglobin concentration in adults with cyanosis from congenital heart disease. Am J Cardiol 2011; 107: 595-599.

13 Oechslin E. Hematological management of the cyanotic adult with congenital heart disease. Int J Cardiol 2004; 97: Suppl. 1, 109-115

14 Van De Bruaene A, Delcroix M, Pasquet A, et al. Iron deficiency is associated with adverse outcome in Eisenmenger patients. Eur Heart J 2011; 32: 2790-2799.

15 Dimopoulos K, Giannakoulas G, Wort SJ, et al. Pulmonary arterial hypertension in adults with congenital heart disease: distinct differences from other causes of pulmonary arterial hypertension and management implications. Curr Opin Cardiol 2008; 23: 545-554.

16 Kaemmerer H, Fratz S, Braun SL, et al. Erythrocyte indexes, iron metabolism, and hyperhomocysteinemia in adults with cyanotic congenital cardiac disease. Am J Cardiol 2004; 94: 825-828. 
Ammash N, Warnes CA. Cerebrovascular events in adult patients with cyanotic congenital heart disease. IAm Coll Cardiol 1996; 28: 768-772.

18 Tay ELW, Peset A, Papaphylactou M, et al. Replacement therapy for iron deficiency improves exercise capacity and quality of life in patients with cyanotic congenital heart disease and/or the Eisenmenger syndrome. Int J Cardiol 2011; 151: 307-312.

19 Diller GP, Alonso-Gonzalez R, Kempny A, et al. B-type natriuretic peptide concentrations in contemporary Eisenmenger syndrome patients: predictive value and response to disease targeting therapy. Heart 2012; 98: 736-742.

20 Diller GP, Dimopoulos K, Okonko D, et al. Exercise intolerance in adult congenital heart disease: comparative severity, correlates, and prognostic implication. Circulation 2005; 112: 828-835.

21 Dimopoulos K, Okonko DO, Diller GP, et al. Abnormal ventilatory response to exercise in adults with congenital heart disease relates to cyanosis and predicts survival. Circulation 2006; 113: 2796-2802.

22 Raphael C, Briscoe C, Davies J, et al. Limitations of the New York Heart Association functional classification system and self-reported walking distances in chronic heart failure. Heart 2007; 93: 476-482.

23 Kempny A, Dimopoulos K, Alonso-Gonzalez R, et al. Six-minute walk test distance and resting oxygen saturations but not functional class predict outcome in adult patients with Eisenmenger syndrome. Int J Cardiol 2013 [in press DOI: 10.1016/j.ijcard.2013.07.227].

24 Moceri P, Dimopoulos K, Liodakis E, et al. Echocardiographic predictors of outcome in Eisenmenger syndrome. Circulation 2012; 126: 1461-1468.

25 Galiè N, Beghetti M, Gatzoulis MA, et al. Bosentan therapy in patients with Eisenmenger syndrome: a multicenter, double-blind, randomized, placebo-controlled study. Circulation 2006; 114: 48-54.

26 Gatzoulis MA, Beghetti M, Galiè N, et al. Longer-term bosentan therapy improves functional capacity in Eisenmenger syndrome: results of the BREATHE-5 open-label extension study. Int J Cardiol 2008; 127: 27-32

27 Berger RMF, Beghetti M, Galiè N, et al. Atrial septal defects versus ventricular septal defects in BREATHE-5, a placebo-controlled study of pulmonary arterial hypertension related to Eisenmenger's syndrome: a subgroup analysis. Int J Cardiol 2010; 144: 373-378.

28 Mukhopadhyay S, Nathani S, Yusuf J, et al. Clinical efficacy of phosphodiesterase-5 inhibitor tadalafil in Eisenmenger syndrome - a randomized, placebo-controlled, double-blind crossover study. Congenit Heart Dis 2011; 6: 424-431.

29 Chau EMC, Fan KYY, Chow WH. Effects of chronic sildenafil in patients with Eisenmenger syndrome versus idiopathic pulmonary arterial hypertension. Int J Cardiol 2007; 120: 301-305.

30 Iversen K, Jensen AS, Jensen TV, et al. Combination therapy with bosentan and sildenafil in Eisenmenger syndrome: a randomized, placebo-controlled, double-blinded trial. Eur Heart J 2010; 31: 1124-1131.

31 Diller GP, Alonso-Gonzalez R, Dimopoulos K, et al. Disease targeting therapies in patients with Eisenmenger syndrome: response to treatment and long-term efficiency. Int J Cardiol 2013; 167: 840-847.

32 Dimopoulos K, Inuzuka R, Goletto S, et al. Improved survival among patients with Eisenmenger syndrome receiving advanced therapy for pulmonary arterial hypertension. Circulation 2010; 121: 20-25.

33 D'Alto M, Romeo E, Argiento P, et al. Therapy for pulmonary arterial hypertension due to congenital heart disease and Down's syndrome. Int J Cardiol 2013; 164: 323-326.

34 Duffels MGJ, Vis JC, van Loon RLE, et al. Down patients with Eisenmenger syndrome: is bosentan treatment an option? Int J Cardiol 2009; 134: 378-383.

35 Habib G, Hoen B, Tornos P, et al. Guidelines on the prevention, diagnosis, and treatment of infective endocarditis (new version 2009): the Task Force on the Prevention, Diagnosis, and Treatment of Infective Endocarditis of the European Society of Cardiology (ESC). Eur Heart J 2009; 30: 2369-2413.

36 Daliento L, Somerville J, Presbitero P, et al. Eisenmenger syndrome. Factors relating to deterioration and death. Eur Heart J 1998; 19: 1845-1855.

37 Sandoval J, Santos LE, Córdova J, et al. Does anticoagulation in Eisenmenger syndrome impact long-term survival? Congenit Heart Dis 2012; 7: 268-276.

38 European Society of Gynecology (ESG), Association for European Paediatric Cardiology (AEPC), German Society for Gender Medicine (DGesGM), et al. ESC Guidelines on the management of cardiovascular diseases during pregnancy: the Task Force on the Management of Cardiovascular Diseases during Pregnancy of the European Society of Cardiology (ESC). Eur Heart J 2011; 32: 3147-3197.

39 Broberg CS, Uebing A, Cuomo L, et al. Adult patients with Eisenmenger syndrome report flying safely on commercial airlines. Heart 2007; 93: 1599-1603. 